Citation: Carr, S., dasNair, R., Schwartz, A., \& Lincoln, N.B. (2014). Group Memory

Rehabilitation for People with Multiple Sclerosis: A pilot randomised controlled trial. Clinical

Rehabilitation, 28(6), 552-561. DOI : 10.1177/0269215513512336

\title{
Group memory rehabilitation for people with multiple sclerosis: a feasibility randomized controlled trial
}

\author{
Sara E Carr', Roshan das Nair ${ }^{2}$, Annette F Schwartz' \\ and Nadina B Lincoln ${ }^{2}$
}

\begin{abstract}
Objective: To assess the feasibility and effectiveness of a group memory rehabilitation programme combining compensation and restitution strategies.

Design: Randomized controlled trial.

Setting: Community.

Participants: People with multiple sclerosis who reported memory difficulties were recruited.

Interventions: A group memory rehabilitation programme, comprising ten 1.5-hour sessions, was compared with a waiting list control.

Main measures: The primary outcome was the Everyday Memory Questionnaire. Secondary outcomes included the General Health Questionnaire 28 and MS Impact Scale administered four and eight months after randomization. In addition, those in the intervention group gave feedback about the intervention. Results: Forty-eight participants were recruited. They were aged 34-72 years (mean 54.3, SD 11.0) and $33(69 \%)$ were women. There were no significant differences between the two groups on the Everyday Memory Questionnaire or MS Impact Scale $(P>0.05)$ at four or eight months after randomization. However, the intervention group reported significantly better mood than controls on the GHQ-28 at eight months $(P=0.04)$. Participants showed minimal benefit from the memory rehabilitation programme on quantitative measures but the intervention was well received, as indicated by positive feedback at the end of the intervention.

Conclusions: There was no significant effect of the intervention on memory but there was a significant effect on mood. The results suggest a larger scale study is justified.
\end{abstract}

\section{Keywords}

Multiple sclerosis, memory, rehabilitation, groups, cognition 


\section{Introduction}

Cognitive deficits are common in people with multiple sclerosis, with approximately $40-65 \%$ of patients with multiple sclerosis developing varying degrees of cognitive dysfunction. ${ }^{1,2}$ This includes impairments of attention, memory and executive function. Memory problems are not only persistent but are also incapacitating, difficult to treat and often associated with frustration, distress and mood disorders for the patients and their partners or carers. ${ }^{3}$

Memory rehabilitation is a structured set of therapeutic activities designed to enhance everyday memory functioning by increasing individuals' knowledge of memory impairments, offering strategies to enable better management of specific difficulties, and retraining individuals' ability to organize and retrieve information. Memory rehabilitation has been provided for people with multiple sclerosis withpromisingresults. ${ }^{4-11}$ Interventions have mainly involved individual training on computerized tasks, ${ }^{5,7-11}$ but some have delivered face to face group sessions. ${ }^{6}$

Although the findings from individual studies mostly appear promising, reviews of memory rehabilitation in people with multiple sclerosis have indicated that the effectiveness of memory rehabilitation programmes is far from conclusive. O'Brien et al. ${ }^{12}$ reviewed cognitive interventions for people with multiple sclerosis, and highlighted the need for 'more methodologically rigorous research' (p. 761). A Cochrane review ${ }^{13}$ of neuropsychological rehabilitation for people with multiple sclerosis identified 14 studies with 770 patients and concluded there was low-level evidence that neuropsychologi-cal rehabilitation reduced cognitive symptoms in people with multiple sclerosis, but there was no evidence of an effect on cognitive or emotional functions. Das Nair et al. ${ }^{4}$ conducted a Cochrane review of randomized controlled trials of memory rehabilitation for people with multiple sclerosis and also found no evidence to support or refute the effectiveness of memory rehabilitation on memory function or other functional abilities, either immediately or in the long term.

One of the trials included in the systematic review ${ }^{4}$ was a study by das Nair and Lincoln. ${ }^{6}$
Seventy-two participants with memory problems following stroke $(n=17)$, traumatic brain injury ( $n$ $=16)$ and multiple sclerosis $(n=39)$ were randomly allocated to one of three conditions: restitution, compensation or self-help (control group). The restitution and compensation programmes both included teaching internal memory strategies and errorless learning, while the compensation group were also taught how to use external memory aids. The self-help group were only taught relaxation techniques and coping strategies. There were no significant differences in outcomes on the primary outcome measure, the Everyday Memory Questionnaire. However, both the compensation and restitution groups used significantly more internal memory strategies compared to the selfhelp group and the compensation group used significantly more external memory aids at seven months after randomization compared to the selfhelp group. Das Nair and Lincoln ${ }^{6}$ commented that although they compared restitution and compensation approaches, in clinical practice these two approaches are typically used together.

The aim of the present study was to assess the feasibility of delivering a group memory rehabilitation programme which combined the restitution and compensation strategies previously evaluated separately by das Nair and Lincoln. ${ }^{6}$ The effectiveness of the programme was evaluated with the aim of developing a multicentre randomized trial.

\section{Method}

Ethical approval was obtained from the National Research Ethics Service, South London, and research and development approval was obtained from Central Surrey Health.

Potential patients with multiple sclerosis were identified from a register of patients who attended Central Surrey Health MS clinics. They were invited to take part in the study by letter, which contained information on the purpose of the study, including the focus on memory problems in daily life, and what participation would involve. Patients who were interested were asked to contact one of the researchers or to complete the consent form and return it in a pre-stamped envelope. The patients 
who gave informed consent were then contacted to arrange a baseline assessment with an assistant psychologist, who worked under the supervision of a consultant clinical neuropsychologist. The Wechsler Memory Scale-IV (WMS-IV) ${ }^{15}$ was completed to assess for memory impairment. Individuals were included if they reported memory problems in daily life, were more than 12 months since diagnosis, able to give informed consent, able to speak and understand conversational English, and able to attend the outpatient unit where the treatment sessions were delivered. Patients with very severe memory problems who were considered by the consultant clinical psychologist or multiple sclerosis specialist nurse to not be able to cope with group sessions were excluded. Patients also gave consent for treatment sessions to be video-recorded.

After recruitment, participants were asked to complete further baseline measures at home and return by post: These were:

- Guy's Neurological Disability Scale (GNDS), ${ }^{16}$ a measure of 12 impairments due to multiple sclerosis, including psychological aspects (memory and concentration, mood) and physical aspects (mobility). The optional question on sexual function was not included.

- Everyday Memory Questionnaire (EMQ-28), ${ }^{17}$ a measure of self-reported memory difficulties. Two versions were completed: a patient version and a carer version, the latter completed by someone who knew the patient well, such as a carer, partner, close friend or relative. Each item was scored from 0 to 5 according to the frequency of problems reported. High scores indicate more memory problems.

- The General Health Questionnaire 28 (GHQ28), ${ }^{18}$ a measure of psychological wellbeing. Participants were asked to rate how they had been in general over the past few weeks. The GHQ-28 was scored using a Likert scale from 0 to 3 for each item. High scores indicate greater distress.

- The Multiple Sclerosis Impact Scale (MSIS29), ${ }^{19}$ a disease-specific quality-of-life scale, which measures the physical and psychological impact of multiple sclerosis. Each item was rated using a 5-point Likert-type scale from 1 (not at all bothered) to 5 (extremely bothered). Scores range from 29 to 145 , with high scores indicating greater impact of the disease.

Participants were randomly allocated to either a treatment group or a control group using a computergenerated list prepared in advance of the study and held by an independent researcher at the University of Nottingham. Participants randomized to the treatment group were invited to attend a group intervention programme consisting of ten 1.5-hour sessions and homework over a period of 10 weeks. Eight participants were invited to attend each group and received treatment together for the ten sessions. If sessions were missed, for example due to illness, then participants were invited to attend the next session early in order to catch up on the missed material. The programme was based on the group programmes previously evaluated, ${ }^{6,20}$ but both restitution and compensation strategies were included for all participants. The programme included: one introductory session; three sessions on attention training; three sessions on internal memory strategies; two sessions on external memory aids; and one concluding session to bring together everything that had been learned and to reflect on the best strategies for each individual. Homework was recommended at the end of each session. An assistant psychologist delivered the treatment groups based entirely on the manual (available from the corresponding author). Group sessions were video-recorded to check that sessions corresponded with the manual. Participants completed a feedback form during the final session to assess the benefit of the sessions and to give participants the opportunity to suggest improvements to the programme. Participants' travel was reimbursed at the end of the sessions.

Participants randomly allocated to the control group received their usual care and all other rehabilitation (e.g. physiotherapy, occupational therapy) continued as usual. They were offered the opportunity to attend the memory rehabilitation programme once all eight-month outcomes had been completed.

Outcome assessments were carried out four and eight months after random allocation. The Everyday 
Memory Questionnaire patient version at four months was the primary outcome measure. Secondary outcome measures included the Everyday Memory Questionnaire patient version at eight months and the GHQ-28, MSIS-29 and Everyday Memory Questionnaire carer version at four and eight months. Outcome assessments were posted to participants' homes and returned to the University of Nottingham, where they were scored by a researcher blind to the group allocation. If participants failed to return the outcome questionnaires, they were telephoned once and any missing items were completed by telephone. For those questionnaires where items were missed out, if these occurred for less than $10 \%$ of questions in a questionnaire, the missing item was replaced with the mean for the questionnaire. As this was a pilot study to assess the feasibility of the intervention, a priori sample size calculations were not conducted. An intention-to-treat analysis was used.

Results were analysed using SPSS version 19. As the scales were ordinal and the sample size small, non-parametric statistics were used. The feedback questionnaires were summarized in a table and the free text comments were analysed using content analysis. ${ }^{21}$

\section{Results}

Between January and May 2011, 362 patients were referred to the study (Figure 1). Fifty-seven (16\%) agreed to participate. Of these, one declined due to transport problems and one was excluded because of severe memory difficulties and because it was considered that a group setting would not be suitable. The first 48 who met the inclusion criteria were recruited. The remaining seven participants were offered the memory rehabilitation programme once the study had been completed and were not included in the evaluation.

The demographic characteristics and baseline measures for each group are summarized in Table 1.

The average intervention group attendance was 7.9 sessions out of 10 (SD 0.23). Two participants dropped out of the intervention prior to the start of the programme due to illness and time constraints.
A further two participants began the group sessions but left after session 2: one participant would have preferred individual memory rehabilitation and the other had personal problems.

The groups were comparable at baseline. Differences in the primary and secondary outcome measures at four and eight months post randomization were compared between groups using independent-samples Mann-Whitney $\boldsymbol{U}$ tests. Medians are shown in Table 2 to summarize the analysis. Means and standard deviations for each of the measures are also shown in Table 2 to allow inclusion of the study in future meta-analyses. Missing items were replaced for six participants.

At four months and eight months, there were no significant differences between the groups on the the EMQ self-report (four months $\boldsymbol{P}=0.77$, eight months $\boldsymbol{P}=0.13$ ). There was a significant difference in mood at eight months on the GHQ $(\boldsymbol{P}=0.04)$ but not on other secondary measures. Results are shown in Table 2. In order to assess the effect over time, an area under the curve corrected for baseline was calculated for each participant. Comparison of the area under the curve between the two groups showed no significant differences $(\boldsymbol{P}>0.05)$. Results are also shown in Table 2 .

The results from the questionnaires distributed to the intervention group during the final session, are shown in Table 3. All 18 of the 24 intervention group participants who completed the feedback questionnaire 'agreed' or 'strongly agreed' that the programme facilitated the reflection on their own memory difficulties, offered them the opportunity to learn from others' experiences, and gave them a set of tools or strategies that they could use to deal with the memory difficulties.

Free text comments written on the intervention feedback forms were generally positive. Fifteen out of 18 participants who completed the feedback questionnaire stated that they felt that attending the memory rehabilitation programme had made a difference to how they coped with memory difficulties, with participants giving reasons related to being able to use specific strategies, improving their confidence and not feeling like the only one 


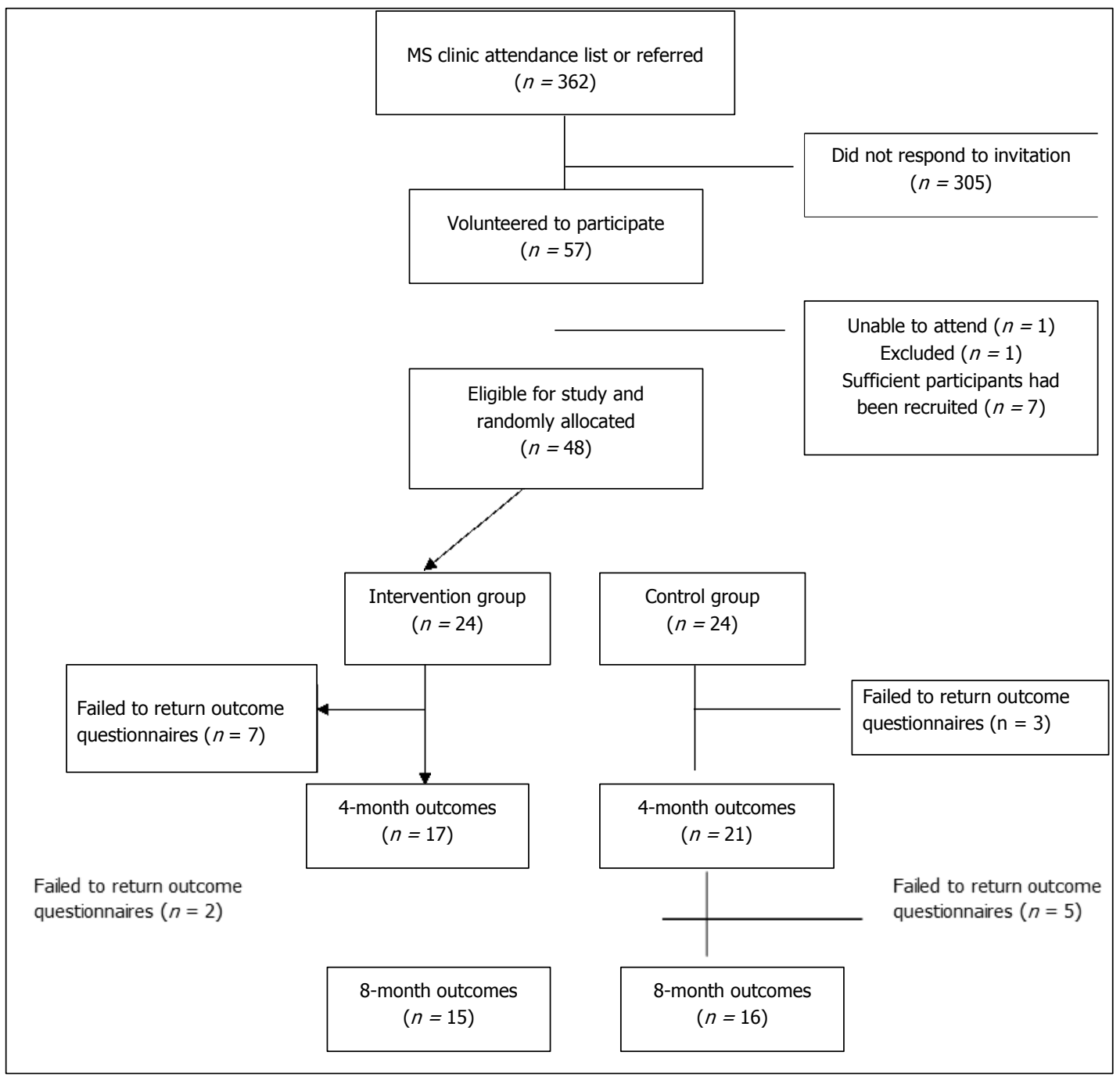

Figure 1. Recruitment and patient follow-up.

with memory difficulties. For example, one participant stated:

Simply being with other people who talked about their experiences has shown me that it's not just me, that actually my memory is a lot better than many people's and [the programme] has taught me to get less stressed when I forget things.

Three other participants were unsure about the influence of the programme on the way they coped with memory difficulties, with comments similar to:
Not yet. I missed four sessions and in any case [I] need to read, assimilate and practice techniques.

All 17 participants who commented on whether they felt the memory programme would be beneficial to other people who experience memory difficulties were strongly in favour of the programme, with comments such as:

Very beneficial - I have been telling my friends about some of the strategies already. 
Table 1. Demographic characteristics and baseline measures of the participants.

\begin{tabular}{|c|c|c|c|c|c|c|}
\hline \multirow[t]{2}{*}{ Demographic characteristics } & \multicolumn{3}{|c|}{ Intervention group $(n=24)$} & \multicolumn{2}{|c|}{ Control group $(n=24)$} & \multirow[b]{2}{*}{ SD } \\
\hline & $n$ & Mean & SD & $n$ & Mean & \\
\hline Age & 24 & 55.8 & 10.2 & 24 & 52.9 & 11.8 \\
\hline Years of education & 24 & 15.7 & 3.6 & 24 & 13.5 & 3.4 \\
\hline Years since diagnosis & 24 & 16.3 & 11.3 & 24 & 12.3 & 9.1 \\
\hline \multicolumn{7}{|l|}{ Gender $(n(\%))$} \\
\hline Men & $7(29)$ & & & $8(33)$ & & \\
\hline Women & $17(71)$ & & & $16(67)$ & & \\
\hline \multicolumn{7}{|l|}{ Type of multiple sclerosis $(n(\%))$} \\
\hline Primary progressive & $6(25)$ & & & $10(42)$ & & \\
\hline Secondary progressive & $4(17)$ & & & $4(17)$ & & \\
\hline Relapsing remitting & $7(29)$ & & & $9(37)$ & & \\
\hline Benign & $2(8)$ & & & 0 & & \\
\hline Unknown & $5(21)$ & & & $1(4)$ & & \\
\hline \multicolumn{7}{|l|}{ Baseline measures } \\
\hline \multicolumn{7}{|l|}{ Wechsler Memory Scale-IVa } \\
\hline Auditory memory & 23 & 95.8 & 17.5 & 23 & 100.7 & 17.4 \\
\hline Visual memory & 19 & 97.5 & 12.5 & 21 & 98.3 & 17.4 \\
\hline Visual working memory & 16 & 97.3 & 17.3 & 16 & 99.3 & 15.9 \\
\hline Immediate memory & 20 & 97.5 & 14.2 & 21 & 100.2 & 19.1 \\
\hline Delayed memory & 20 & 98.4 & 15.5 & 21 & 100.9 & 18.1 \\
\hline EMQ - self-report & 24 & 27.3 & 21.6 & 24 & 30.0 & 22.6 \\
\hline EMQ - carer report & 24 & 21.5 & 19.5 & 24 & 15.8 & 17.0 \\
\hline GHQ-28 & 24 & 23.5 & 9.8 & 24 & 25.0 & 9.0 \\
\hline MS Impact Scale & 24 & 66.7 & 23.6 & 24 & 76.0 & 24.7 \\
\hline Guys Neurological Disability Scale & 24 & 16.2 & 7.9 & 24 & 15.54 & 6.73 \\
\hline
\end{tabular}

GHQ-28, General Health Questionnaire 28; EMQ, Everyday Memory Questionnaire.

alt was not possible to assess all participants on every index of the WMS-IV. Twelve participants completed the Older Adults Battery which does not assess Visual Working Memory; four participants had vision/motor difficulties and so some tests could not be completed; one participant was recently assessed on memory at another centre so they were not re-assessed for the present study; and one participant was becoming distressed with the WMS-IV so the test was discontinued.

and:

Yes - because it helps you understand how memory works and develop coping mechanisms

Participants were also asked about the improvements that they felt needed to be made to the programme for future memory groups. Six out of 15 participants who answered this question stated that no improvements were necessary. Some participants commented on the practicalities of running the groups; for instance, three individuals remarked that a bigger room was needed to better accommodate people using mobility aids; two participants stated that the facilitator needed to consider how the group is constructed in terms of which individuals are selected to work together; one participant stated that the group sessions were too lengthy and could be reduced. One participant commented on the content of the manual and would have liked more discussion about internal memory aids. Two participants also stated that they would have liked a formal assessment of memory at the end of the study. All participants in both the intervention and control groups were given a brief written neuropsychological report on their memory 
scores after the study, which they felt was beneficial.

Finally, many other comments were offered by participants in the intervention group on the feedback forms and in writing after the programme had been completed. These comments highlight the usefulness of the programme:

This course has made me feel more confidence and positive and encouraged me to employ lots of different ideas.

I was very nervous about taking part in this programme but as soon as the first session had begun I was put at ease and found the whole process very positive.

Attending your course made me stop, think, adopt some strategies suggested and feel smug that I was already practising some ideas that I had 'actually thought of myself!'It really helped, calmed me down, made me face the issues and therefore deal with them.

\section{Discussion}

This randomized trial of a group memory rehabilitation programme showed no statistically significant differences between the intervention and control groups on the Everyday Memory Questionnaire. The intervention group reported fewer everyday memory difficulties at eight months compared to the control group, and the Everyday Memory Questionnaire - carer version mirrored these findings. However, these were not statistically significant differences. There were significant differences between groups in mood at eight months after randomization. The beneficial effect of the intervention on mood could be because the participants in the intervention group were less distressed by their memory problems as a result of the memory rehabilitation or due to the social interaction in a group setting. In addition there was no significant effect over time when are under the curve analysis was used. This may reflect an initial deterioration in abilities as participants become aware of their problems, which is followed by an improvement as they learn to cope. These results suggest there may be some benefits of the 
Table 3. Responses of the intervention group participants during the final session of the memory rehabilitation programme.

\begin{tabular}{|c|c|c|c|c|c|c|c|c|c|c|}
\hline \multirow[t]{2}{*}{ Question } & \multicolumn{2}{|c|}{$\begin{array}{l}\text { Strongly } \\
\text { agree }\end{array}$} & \multicolumn{2}{|c|}{ Agree } & \multicolumn{2}{|c|}{$\begin{array}{l}\text { Don't } \\
\text { know }\end{array}$} & \multicolumn{2}{|c|}{ Disagree } & \multicolumn{2}{|c|}{$\begin{array}{l}\text { Strongly } \\
\text { disagree }\end{array}$} \\
\hline & $n$ & $(\%)$ & $n$ & $(\%)$ & $n$ & $(\%)$ & $n$ & $(\%)$ & $n$ & $(\%)$ \\
\hline $\begin{array}{l}\text { The memory sessions gave me an } \\
\text { opportunity to reflect on memory difficulties } \\
\text { I have been experiencing and how I have } \\
\text { coped so fara }\end{array}$ & 8 & $(47)$ & 9 & (53) & 0 & (0) & 0 & (0) & 0 & (0) \\
\hline $\begin{array}{l}\text { The memory sessions introduced me to a set } \\
\text { of tools I can use to help me remember things } \\
\text { better }\end{array}$ & 8 & (44) & 10 & $(56)$ & 0 & (0) & 0 & (0) & 0 & (0) \\
\hline $\begin{array}{l}\text { I do not feel any more confident in dealing } \\
\text { with my memory difficulties }\end{array}$ & 1 & (6) & 0 & (0) & 5 & (28) & 8 & (44) & 4 & (22) \\
\hline $\begin{array}{l}\text { The memory sessions gave me an } \\
\text { opportunity to discuss my memory problems } \\
\text { with others and learn from others' } \\
\text { experiences }\end{array}$ & 13 & (72) & 5 & (28) & 0 & (0) & 0 & (0) & 0 & (0) \\
\hline
\end{tabular}

aOne participant failed to answer this question $(n=17)$.

intervention, but it is not clear what led to the improvement. Given the small sample size this could also be a chance finding.

The lack of differences on the Everyday Memory Questionnaire may be because the participants had little chance to put into practice what they had learned during the programme. However, it may also reflect the scoring of the Everyday Memory Questionnaire. The Everyday Memory Questionnaire only records frequency of memory failure in daily life, but it may be more useful to know the extent to which the item causes a problem in daily life. This was also noted to be a problem in previous research, ${ }^{6}$ but the Everyday Memory Questionnaire was used as there was no appropriate alternative available with good psychometric properties. Recent studies have included a rating of the importance of the activity in addition to the frequency, ${ }^{22}$ but the acceptability of this modification was not known at the time the study was designed.

The lack of significant difference may also have occurred as the sample size was small and there was some drop-out, so that only $65 \%$ of participants completed the eight-month outcomes, as shown in Figure 1. The reason the sample size was small was that funding was provided for a small-scale pilot study to test the feasibility of the combined intervention and to provide the data to enable a power calculation to be conducted for a definitive study. A post hoc power calculation on the Everyday Memory Questionnaire indicated that with $80 \%$ power and a significance level of 0.05 , it would have been necessary to recruit 46 participants in each group to achieve a statistically significant difference. Only $16 \%$ of the individuals approached for the study volunteered to participate. This is probably partly because some did not have memory problems. All patients with multiple sclerosis who were known to a community rehabilitation service were contacted, as there was no means of identifying beforehand those with cognitive problems. It may be that the majority did not feel they had memory problems in daily life, but this figure is lower than expected from previous studies of the frequency memory impairment. ${ }^{2}$ It may also reflect the recruitment source. Participants were recruited from a community rehabilitation team. They may therefore have felt that they had already received sufficient rehabilitation for any cognitive problems. 
The study showed the feasibility of running the combined restitution and compensation memory rehabilitation programme. In general, participants who completed feedback questionnaires viewed the intervention favourably and reported the short-and long-term benefits of memory rehabilitation. Many participants reported increased confidence in dealing with memory difficulties and better memory functioning. This is consistent with qualitative feedback from a previous trial of compensation and restitution strategies. ${ }^{14}$ The assistant psychologist who facilitated the programme identified that there was general consensus that the programme was clearly set out and the majority of the exercises were useful and could be integrated into everyday life. In the case where some exercises did not suit the group format or were not well received by participants, the manual was further refined. A group facilitator training manual was also developed for future facilitators for consistency in running the groups. Although group attendance was recorded, there was no recording of homework completion, which would have allowed monitoring of the integration of the memory activities into everyday life and may indicate adherence to the programme. While everyone in the intervention groups was able to participate, a minority felt that the pace of the sessions was not quite right. Matching approaches to individual learning should be an important consideration for future programmes. Future research should also examine grouping individuals with similar memory difficulties together, so they can get the most out of each session.

The outcomes did not include a standardized test of memory. Memory rehabilitation is not necessarily expected to improve memory impairment but the ability to cope. However, inclusion of a standardized test at outcome assessment would have indicated whether there were changes in the underlying memory impairment. Most previous research on memory rehabilitation has included memory tests as outcome measures, ${ }^{5,-11}$ but as the focus of the intervention was on coping with memory problems in daily life, self-reported problems in daily life were assessed. Few previous studies have included longterm follow-up. As multiple sclerosis is a degenerative condition, cognitive problems are likely to worsen, and therefore it might be anticipated that teaching people to cope will produce more enduring effects than attempting to change the underlying cognitive deficit.

The study was not limited to individuals with memory difficulties on formal testing, and therefore the perception of memory problems may have been related to mood. Mean scores on the Wechsler Memory Scale IV were within the average range, but for some people this level may represent impaired functioning relative to their pre-morbid level and also some people may have had selective memory impairments, which were not evident in the index scores, which are composite scores on several subtests. The researchers felt that at this stage of the investigations, anyone who reported memory difficulties could participate, and the information could be used to refine the selection criteria for future studies. All participants were given a brief summary of the baseline assessment after the study and subjective reports indicated that many individuals valued having had a memory difficulty recognized.

In summary, the effect of a group memory rehabilitation programme in reducing memory problems in daily life remains inconclusive. The quantitative results indicated that there were no significant differences between treatment and control groups on everyday memory function, but the qualitative data suggested some benefits of the intervention. The quantitative data, however, did suggest a beneficial effect on mood. The results are sufficiently encouraging to suggest that a fully powered randomized trial is required to assess the effectiveness and cost-effectiveness of the programme for people with multiple sclerosis.

\section{Clinical messages}

- A group memory rehabilitation programme combining restitution and compensation strategies was feasible with people with multiple sclerosis.

- There was no evidence that the rehabilitation programme produced an improvement in memory abilities in daily life. 
- The participants who attended the group memory rehabilitation programme had less psychological distress at eight months after randomization, suggesting beneficial effect on mood.

- The qualitative feedback from participants who attended the intervention was positive.

\section{Acknowledgements}

We would like to express our thanks to Liz Wilkinson, MS specialist nurse at Central Surrey Health, for her involvement in the study and identifying individuals to participate. We also thank all the participants for their involvement in the study.

\section{Conflict of interest}

The author declares that there is no conflict of interest.

\section{Funding}

The research was supported by a research grant from Biogen Idec Limited, Maidenhead, Berkshire.

\section{References}

1. Calabrese M, Rinaldi F, Grossi P and Gallo P. Cortical pathology and cognitive impairment in multiple sclerosis. Exp Rev Neurother 2011; 11: 425-432.

2. Prakash RS, Snook EM, Lewis J, Motl RW and Kramer AF. Cognitive impairments in relapsing remitting multiple sclerosis: a meta-analysis. Mult Scler 2008; 14 : 1250- 1261.

3. Feinstein A. Mood disorders in multiple sclerosis and the effects on cognition. J Neurol Sci 2006; 245: 63-66.

4. das Nair R, Ferguson H, Stark DL and Lincoln NB. Memory rehabilitation for people with multiple sclerosis. Cochrane Database Syst Rev 2012; 3, CD008754.

5. Chiaravalotti ND, DeLuca J, Moore NB and Ricker JH. Treating learning impairments improves memory performance in multiple sclerosis: a randomized clinical trial. Mult Scler 2005; 11: 58-68.

6. das Nair R and Lincoln NB. Evaluation of rehabilitation of memory in people with neurological disabilities (ReMiND): a randomized controlled trial. Clin Rehabil 2012; 26: 894-903.
7. Hildebrandt H, Lanz M, Hahn HK, et al. Cognitive training in MS: effects and relation to brain atrophy. Restor Neurol Neurosci 2007; 25: 33-43.

8. Allen DN, Goldstein G, Heyman RA and Rondinelli T. Teaching memory strategies to persons with multiple sclerosis. J Rehabil Res Dev 1998; 35: 405-410.

9. Mendozzi L, Pugnetti L, Motta A, Barbieri E, Gambini A and Cazzullo CL. Computer-assisted memory retraining of patients with multiple sclerosis. Ital J Neurol Sci 1998; 19, S431-S438.

10. Solari A, Motta A, Mendozzi L, et al. Computer-aided retraining of memory and attention in persons with multiple sclerosis: a randomized, double-blind controlled trial. J Neurol Sci 2004; 222: 99-104.

11. Tesar N, Bandion K and Baumhackl U. Efficacy of a neuropsychological training programme for patients with multiple sclerosis - a randomised controlled trial. Wiener Klin Wochenschr 2005; 117: 747-754.

12. O'Brien AR, Chiaravalotti N, Goverover Y and DeLuca J. Evidenced-based cognitive rehabilitation for persons with multiple sclerosis: a review of the literature. Arch Phys Med Rehabil 2008; 89: 761-769.

13. Rosti-OtajärviEMandHämäläinenPI.Neuropsychological rehabilitation for multiple sclerosis. Cochrane Database Syst Rev 2011; 11: CD009131.

14. das Nair R and Lincoln NB. The effectiveness of memory rehabilitation following brain damage: a qualitative inquiry of patient perspectives. Neuropsychol Rehabil 2013; 23: 528-545.

15. Wechsler D. Wechsler Memory Scale - fourth edition: technical and interpretive manual. San Antonio, TX: Psychological Corporation, 2009.

16. Sharrack B and Hughes RA. The Guy's Neurological Disability Scale (GNDS): a new disability measure for multiple sclerosis. Mult Scler 1999; 5, 223-233.

17. Sunderland A, Harris JE and Baddeley AD. Do laboratory tests predict everyday memory? A neuropsychological study. J Verbal Learning Verbal Behav 1983; 22: 341-357.

18. Goldberg D. Manual of the General Health Questionnaire. Windsor, UK: NFER Publishing, 1998.

19. Hobart J, Lamping D, Fitzpatrick R, Riazi A and Thompson A. The Multiple Sclerosis Impact Scale (MSIS-29): a new patient-based outcome measure. Brain 2001; 124: 962-973.

20. das Nair R. Effectiveness of memory rehabilitation following brain damage, Vol 2. PhD thesis submitted to the University of Nottingham, 2007.

21. Cavanagh S. Content analysis: concepts, methods and applications. Nurse Res 1997; 4: 5-16.

22. Rehabilitation of memory following traumatic brain injury (ReMemBrIn), 2012. http://www.controlledtrials. com/ISRCTN65792154. 